\title{
N-glycosylation regulates photosynthetic efficiency of Arabidopsis thaliana
}

\author{
Q.-S. JIAO, G.-T. NIU, F.-F. WANG, J.-Y. DONG, T.-S. CHEN, C.-F. ZHOU, and Z. HONG ${ }^{+}$ \\ State Key Laboratory of Pharmaceutical Biotechnology, School of Life Sciences, Nanjing University, Nanjing, \\ 210046 Jiangsu, China
}

\begin{abstract}
$\mathrm{N}$-glycosylation is one of the most important protein modifications in eukaryotes. It has been well established that $\mathrm{N}$-glycosylation plays multiple roles in regulating stress tolerance of plants. However, the effects and mechanism of $\mathrm{N}$-glycosylation on photosynthesis have not been well understood. In the present study, an obvious decrease in photosynthetic capacity and dry mass were detected in alg3-3 and cgll-1, two typical mutants in N-glycosylation process. The maximal photochemical efficiency of PSII decreased significantly in cgll-1. The values of effective quantum yield of PSII photochemistry, rate of photosynthetic electron transport through PSII, and photochemical quenching coefficient, which reflected the photochemical efficiency of plants, decreased as well, while the values of quantum yield of nonregulated energy dissipation of PSII showed obvious enhancement, the similar tendency was also observed in alg3-3. Furthermore, we found that $\mathrm{N}$-glycosylation was also required to maintain the stability of a chloroplast-located protein CAH1, which was closely related to photosynthesis. These results suggest that $\mathrm{N}$-glycosylation plays crucial roles in maintaining photosynthetic efficiency.
\end{abstract}

Additional key words: asparagine-linked glycosylation; biomass; carbonic anhydrase; chlorophyll fluorescence.

\section{Introduction}

Asparagine-linked glycosylation (N-glycosylation) is an essential process occurring in the lumen of the endoplasmic reticulum (ER) and the secretory system of eukaryotic organisms (Silberstein and Gilmore 1996, Helenius and Aebi 2001). It is critical for protein folding, quality control, and transport of the secretory proteins, which plays crucial roles in plant adaptive responses to environmental stresses, such as plant immunity (Kang et al. 2015), temperature tolerance (Zhang et al. 2009), and salt sensitivity (Koiwa et al. 2003, Liu et al. 2018). More than one thousand proteins containing different $\mathrm{N}$-glycosylation sites have been found in the model plant Arabidopsis thaliana (Song et al. 2013). As one of the major and most widespread protein posttranslational modifications in eukaryotes, the core processes are evolutionarily strongly conserved.

The initial synthesis of the lipid-linked oligosaccharide occurs on the cytoplasmic side of the ER and is mainly carried out by a series of glycosyltransferases of the ALG (asparagine-linked glycosylation) family (Bickel et al. 2005). The precursor is then transported across the ER membrane by a flippase-like protein for further synthesis to finally form an oligosaccharide containing three molecules of glucose (Glc), two molecules of N-acetylglucosamine
(GlcNAc), and nine molecules of mannose (Man) in the luminal side of the ER (Higgins 1994, Stagljar et al. 1994, Reiss et al. 1996, Larkin and Imperiali 2011, Schoberer et al. 2018). Subsequently, the completed oligosaccharide $\left(\mathrm{Glc}_{3} \mathrm{Man}_{9} \mathrm{GlcNAc}_{2}\right)$ is transferred by the oligosaccharyltransferase (OST) complex onto the asparagine in the Asn-Xaa-Ser/Thr consensus motif (Xaa represents any amino acid except proline) of the nascent polypeptides (Silberstein and Gilmore 1996, Helenius and Aebi 2001). The mannose glycans are gradually trimmed as part of ERQC (ER-associated quality control compartment) system (Aebi et al. 2010). Then, the correctly folded glycoproteins are transported to the Golgi apparatus and further modified by Golgi resident glycosyltransferases and glycosidases for the formation of glycoproteins with mature oligosaccharide (Song et al. 2013, Strasser 2016).

The significance of $\mathrm{N}$-glycosylation modifications in mammals has been well exemplified by the severe congenital disorders resulting from mutations of the N-glycosylation pathway (Schollen et al. 2005). However, no visible change was found in the Arabidopsis $\mathrm{N}$-glycosylation mutant alg3, which is defective in the biosynthesis of the Dol-PP-linked glycans; it seems reasonable to suggest that the glycans in the structure of mannose type were shown to be nonessential (Henquet et al.

\footnotetext{
$\overline{R e c e i v e d} 27$ March 2019, accepted 15 November 2019.

${ }^{+}$Corresponding author; e-mail: zhihong@nju.edu.cn

Abbreviations: ETR - rate of photosynthetic electron transport through PSII; $\mathrm{F}_{\mathrm{v}} / \mathrm{F}_{\mathrm{m}}$ - maximal photochemical efficiency of PSII; $\mathrm{q}_{\mathrm{N}}-$ nonphotochemical quenching coefficient; $\mathrm{q}_{\mathrm{P}}$ - photochemical quenching coefficient; $\mathrm{SD}$ - standard deviation; $\mathrm{Y}_{(\mathrm{NO})}-$ quantum yield of nonregulated energy dissipation of PSII; $\mathrm{Y}_{(\mathrm{NPQ})}$ - quantum yield of regulated energy dissipation of PSII; $\Phi_{\mathrm{PSII}}-$ effective quantum yield of PSII photochemistry.

Acknowledgments: Authors thank members of the Hong laboratory for critical suggestions for this study. This work was supported by the National Natural Science Foundation of China (31322008, 31870263).
} 
2008). Also, another mutation of N-glycosylation pathway in Arabidopsis, the complex glycan less mutant (cgll-1), which completely lacks the complex N-glycans, has also no visible developmental disorders and only shows some changes in salt-stress response (Kang et al. 2008). The phenotype ambiguity of N-glycosylation mutants in 'ideal' greenhouses may be the reason why this process is rarely studied in plants (Zielinska et al. 2012). Nevertheless, the $\mathrm{N}$-glycosylation abnormalities result in unfolded protein response (von Schaewen et al. 1993, Henquet et al. 2008). This reminds us that many unknown abnormalities are still to be discovered. It is well known that photosynthesis in plants and algae occurs in chloroplasts, and this process is the basis of plant growth and development. In addition, most chloroplast-located proteins are synthesized in the ER and then transported to the chloroplast through the secretory pathway. CAH1, alpha-type carbonic anhydrase (EC 4.2.1.1), is one of the few plant proteins known to be targeted to the chloroplast, and which is essential for efficient photosynthesis (Samuelsson and Karlsson 2001, Burén et al. 2011, Kupriyanova et al. 2017). The mature CAH1 harboring multiple glycoforms of N-glycans (Burén et al. 2011) might be a good substrate to study the relation between N-glycosylation and photosynthesis.

It has long been known that chlorophyll (Chl) fluorescence induction kinetics of leaves from plants provides an indicator of plant photosynthetic capacity. The fluorescence parameters are directly related to the energy transport, the photosynthetic carbon dioxide assimilation rate, and the formation of primary photosynthetic sugars in plants (Harbinson et al. 1990, Murchie and Lawson 2013). As rapid and noninvasive technique for detecting subtle differences of leaf metabolism or developmental disorders of the seedlings (Barbagallo et al. 2003), Chl fluorescence induction kinetics has received increasing attention in the fields of plant physiology, molecular biology, and molecular genetics.

In the present study, we analyzed the phenotype of $\mathrm{ALG}_{3}$ and CGL knockout mutant plants. We showed that the mutants grow normally under greenhouse conditions. In order to investigate whether $\mathrm{N}$-glycosylation could affect the photosynthetic capacity, we also examined if chlorophyll fluorescence parameters could reveal the possible alterations in the PSII photochemistry in the alg3-3 and cgl1-1 mutants. Our data showed that the photosynthetic capacity of alg3-3 and cgll-1 decreased to different degrees. Moreover, the underlying molecular mechanisms of photosynthetic capacity changes were also analyzed.

\section{Materials and methods}

Plant materials and growth conditions: Arabidopsis thaliana, ecotype Columbia (Col-0), was the parental line for mutant plants and the generation of transgenic plants. Seeds of alg3-3 (SALK_046061) and cgl1-1 (CS6192) were obtained from the Arabidopsis Biological Resource Center (ABRC) at Ohio State University. The seeds were surface sterilized with $70 \%$ ethanol and then stratified at $4{ }^{\circ} \mathrm{C}$ for $2 \mathrm{~d}$ in darkness. The stratified seeds were placed on plates containing $1 / 2$ MS medium supplemented with $0.3 \%$ gelrite and $1 \%$ sucrose. The plates were then placed for seed germination and plant growth in a growth chamber (MLR$352 \mathrm{H}-\mathrm{PC}$, Panasonic, Japan) at $22^{\circ} \mathrm{C}$ under irradiance of $100 \pm 5 \mu \mathrm{mol}$ (photon) $\mathrm{m}^{-2} \mathrm{~s}^{-1}$ at long-day conditions with a 16-h light/8-h dark cycle, and 50\% relative humidity.

Chl fluorescence parameters were measured using a portable fluorometer (Mini PAM, Walz, Germany), as described previously by Demmig-Adams et al. (1996). The PSII maximal photochemical efficiency $\left(\mathrm{F}_{\mathrm{v}} / \mathrm{F}_{\mathrm{m}}\right)$ was defined as $\left(F_{m}-F_{0}\right) / F_{m}$, where $F_{m}$ is the maximum fluorescence emission from the dark-adapted state measured with a pulse of saturating light, and $\mathrm{F}_{0}$ is the minimal fluorescence from the dark-adapted state. The effective quantum yield of PSII photochemistry $\left(\Phi_{\mathrm{PSII}}\right)$ was defined as $\left(\mathrm{F}_{\mathrm{m}}{ }^{\prime}-\mathrm{F}_{\mathrm{s}}\right) / \mathrm{F}_{\mathrm{m}}{ }^{\prime}$, where $\mathrm{F}_{\mathrm{m}}{ }^{\prime}$ is the maximum fluorescence emission from the lightadapted state measured with a pulse of saturating light and $\mathrm{F}_{\mathrm{s}}$ is the steady-state level of fluorescence emission at the given irradiance. The coefficient of nonphotochemical quenching $\left(\mathrm{q}_{\mathrm{N}}\right)$ was defined as $\left(\mathrm{F}_{\mathrm{m}}-\mathrm{F}_{\mathrm{m}}{ }^{\prime}\right) /\left(\mathrm{F}_{\mathrm{m}}-\mathrm{F}_{0}{ }^{\prime}\right)$, where $\mathrm{F}_{0}{ }^{\prime}$ is minimal fluorescence of the light-adapted state measured with a far-red pulse. The NPQ parameter was defined according to the equation: $N P Q=\left(F_{m}-F_{m}{ }^{\prime}\right) / F_{m}{ }^{\prime}$. The coefficient of photochemical quenching $\left(\mathrm{q}_{\mathrm{P}}\right)$ was defined as $\left(\mathrm{F}_{\mathrm{m}}{ }^{\prime}-\mathrm{F}_{\mathrm{s}}\right) /\left(\mathrm{F}_{\mathrm{m}}{ }^{\prime}-\mathrm{F}_{0}{ }^{\prime}\right)$ according to Lazár $(2015)$. As $\Phi_{\mathrm{PSII}}$ represents the number of electrons transferred per photons absorbed by PSII, the ETR (the rate of photosynthetic electron transport through PSII) can be calculated as $\mathrm{ETR}=\Phi_{\mathrm{PSII}} \times \mathrm{PAR} \times 0.5 \times 0.84$ according to Genty et al. (1989). The quantum yield of nonregulated energy dissipation of PSII $\left(\mathrm{Y}_{(\mathrm{NO})}\right)$ and the quantum yield of regulated energy dissipation of PSII $\left(\mathrm{Y}_{(\mathrm{NPQ})}\right)$ was also defined according to Kramer et al. (2004).

Dry biomass and leaf Chl content: For dry biomass measuring, shoots of 4-week-old plants were detached and oven-dried at $80^{\circ} \mathrm{C}$ for $24 \mathrm{~h}$; the shoots were then weighed separately. The leaf $\mathrm{Chl}$ content was measured spectrophotometrically as described previously with minor modifications (Liang et al. 2017). Fresh leaves were weighed and incubated at room temperature with $10 \mathrm{~mL}$ of solution (acetone: ethanol $=1: 1$ ) for at least $24 \mathrm{~h}$, then clarified by centrifugation for $5 \mathrm{~min}$ at $13,000 \times \mathrm{g}$, while this process was protected from light. The absorbance of the supernatant was measured at wavelengths 645,652 , and $663 \mathrm{~nm}\left(\mathrm{~A}_{645}, \mathrm{~A}_{652}\right.$, and $\left.\mathrm{A}_{663}\right)$ with the microplate reader (FL-600, BioTek, USA). Chl concentration was estimated using following equations: Chl $a\left[\mathrm{mg} \mathrm{g}^{-1}\right]=$ $\left(12.7 \times \mathrm{A}_{663}-2.69 \times \mathrm{A}_{645}\right) \times \mathrm{V} /(1,000 \times \mathrm{W}), \mathrm{Chl} b\left[\mathrm{mg} \mathrm{g}^{-1}\right]=$ $\left(22.9 \times \mathrm{A}_{645}-4.68 \times \mathrm{A}_{663}\right) \times \mathrm{V} /(1,000 \times \mathrm{W})$, total Chl $\left[\mathrm{mg} \mathrm{g}^{-1}\right]=\left(\mathrm{A}_{652} \times \mathrm{V}\right) /(34.5 \times \mathrm{W})$, where $\mathrm{V}$ represents the volume $[\mathrm{mL}]$ of the extracted solution, and $\mathrm{W}$ represents the fresh mass $[\mathrm{g}]$ of the sample.

Identification of the homozygous mutant plants: Genomic DNA was extracted from the leaves as the template for PCR reaction. To identify the genotype of the T-DNA insertion alg3-3 mutant, the DNA was amplified with the forward primer alg3-F1 GATGCAATCCTAGTCGCACT; 
reverse primer alg3-R1 CCTGAGCAGGGTAGACTTCC, and the T-DNA left border primer LBal TGGTTCACGTAGTGGGCCATCG. To identify the genotype of the cgll-1 mutant, the primer cgll-dcaps-F2 TATCAAACTCCCGTTGCTTC, and primer cgll-dcaps-R2 GACAGCTTGATCAGATCGAT were used for PCR amplification. The PCR products were digested with Hinf I (TaKaRa Bio, Japan) and then electrophoresed.

Transgenic plant construct: $p C A H 1: G F P$ was generated from $p P Z P 212-B R I 1$ : BRI1-GFP as follows. The primers 5'-ATGCCTGCAGGTCGACATATGCAGTGCCCATAT TCTGAGG-3'/5'-TTTACTCATGGGATCATTGGGTTTT TTCTTTTTGTTACCG-3' were used to amplify a $4.3-\mathrm{kb}$ genomic fragment consisting of $\approx 1.5 \mathrm{~kb}$ of the $C A H 1$ promoter region, which was inserted via the SalI and BamHI restriction sites. The resulting construct carries a kanamycin-resistant gene as the selectable marker for plant transformation.

Western blot analyses: Arabidopsis seeds were germinated on 1/2 MS medium. To inhibit N-glycosylation, 2-week-old seedlings were transferred to $1 / 2 \mathrm{MS}$ medium containing $30 \mu \mathrm{g} \mathrm{mL}^{-1}$ tunicamycin (Sigma) for continued growth for another $24 \mathrm{~h}$ in the growth chamber as described previously with minor modifications (McCormack et al. 2015). The samples were prepared for loading by adding $2 \times$ sample buffer $[0.125 \mathrm{M}$ Tris $(\mathrm{pH} 6.8), 4.0 \% \mathrm{SDS}$, $20 \%$ glycerol, 0.2 M DTT, $0.02 \%$ bromophenol blue], and heating the samples at $95^{\circ} \mathrm{C}$ for $10 \mathrm{~min}$. Then the supernatants were used for SDS-PAGE on $8.0 \%$ Trisacetate (Liu et al. 2018). Next, proteins in the gel were electrophoretically transferred to PVDF membrane (Roche, Switzerland). Membranes were incubated with primary antibodies in 5.0\% milk at room temperature. The primary antibody used in this study was mouse anti-GFP (Abcam); the secondary antibody was HRP-conjugated goat anti-mouse IgG (D110087, BBI). Rubisco small subunit ( $\mathrm{RbcS}$ ) was used as a loading control. The SDS-PAGE containing $\mathrm{RbcS}$ was stained with Coomassie Brilliant Blue $R-250(0.05 \%$ CBB, $10.0 \%$ acetic acid, and 5.0\% methanol) for $2 \mathrm{~h}$ and then destained by destaing solution (7.0\% acetic acid and 5.0\% methanol). The relative amount of proteins was determined by stained $\mathrm{RbcS}$ bands separated by SDS-PAGE (Dyballa and Metzger 2009).

Statistical analysis: The results were expressed as the mean \pm standard deviation. The data were statistically evaluated with one-way analysis of variance (ANOVA) between the different groups and by Tukey's multiple comparisons test. The difference was considered to be statistically significant when $P \leq 0.05$.

\section{Results}

alg3-3 and cgll-1 showed inconspicuous phenotype under optimal conditions in greenhouse: $\operatorname{alg} 3-3$ is a T-DNA insertion mutant identified in Arabidopsis (Fig. 1A), it derives from incidental splicing of the third extron carrying the T-DNAs, which blocks the first mannose adjunction step on Dol-PP-linked glycans in ER (Fig. 1C). Besides, we also investigated another Arabidopsis cgll-1 mutant that disrupts adding a GlcNAc residue to the $\mathrm{Man}_{5} \mathrm{GlcNAc}_{2}$ glycan in Golgi body (Fig.1 B,C). Both of these homozygous plants did not show any visible changes in their phenotype under normal laboratory conditions (Fig. 1C). Nevertheless, a dry biomass accumulation decreased significantly in the mutant plants compared with the wild type (WT) (Fig. 1S, supplement).

$\mathbf{N}$-glycosylation defects induce changes in $\mathbf{F}_{\mathrm{v}} / \mathbf{F}_{\mathbf{m}}$ : The Chl fluorescence parameter, $F_{v} / F_{m}$, reflects the maximum quantum efficiency of PSII photochemistry in the darkadapted state and it has been widely used for early stress detection in plants (Schansker et al. 2014, Magyar et al. 2018). Our results showed that the value of $F_{v} / F_{m}$ declined differently in the typical N-glycosylation-deficient alg3-3 and cgll-1 mutant plants compared with the WT plants, especially in the cgll-1 mutant, whose values were significantly lower than those in the leaves of WT (Fig. 2). Thus, the above data suggested that their photosynthetic capacity was damaged to a certain degree.

Photochemical efficiency decreased in alg3-3 and cgll-1: In order to investigate further the effects of $\mathrm{N}$-glycosylation on actual photochemical efficiency of plants, correlation parameters for photochemical efficiency were measured and analyzed. The $\Phi_{\text {PSII }}$ denotes the effective quantum yield of PSII photochemistry for the light-adapted state, also denoted as effective quantum yield of PSII photochemistry, which is an indicator of the real photochemical activity (Genty et al. 1989). In the present study, the data clearly showed that the values of $\Phi_{\text {PSII }}$ decreased obviously with the increase of light intensity both in the mutant plants and the WT (Fig. 3A). However, it is worth mentioning that the mutants always exhibited lower $\Phi_{\mathrm{PSII}}$ values than that of the WT under the same irradiance (Fig. 3A). The $\mathrm{q}_{\mathrm{P}}$ (Fig. 3C), which estimates a fraction of 'open' PSII reaction centers based on a puddle model (Stirbet 2013, Lazár 2015), had the same tendency of change as that of the $\Phi_{\text {PSII }}$. Similarly, the photochemical efficiency and the rate of photosynthetic electron transport through PSII (ETR) also showed a downward trend in the mutants (Fig. 3B), compared with that of WT. These changes could give a good indication that the defective of $\mathrm{N}$-glycosylation led to a decrease in the photosynthetic capacity of plants.

Nonphotochemical quenching in alg3-3 and cgll-1: Nonphotochemical quenching parameter (NPQ) equals the ratio of the quantum yield of regulated energy dissipation to the quantum yield of nonregulated energy dissipation of PSII (Lazár 2015). In order to further investigate whether nonphotochemical processes were affected in the mutant plants, the coefficient of nonphotochemical quenching $\left(\mathrm{q}_{\mathrm{N}}\right)$, the NPQ parameter (Fig. 4A,B), and the quantum yield of regulated energy dissipation in PSII, $\mathrm{Y}_{(\mathrm{NPQ})}($ Fig. $4 C$ ), were analyzed. The nonphotochemical processes rose along with the increase of light intensity, slight changes still could be found in $\mathrm{q}_{\mathrm{N}}$ and $\mathrm{Y}_{(\mathrm{NPQ})}$, while the NPQ values were always lower than their counterparts in WT, 

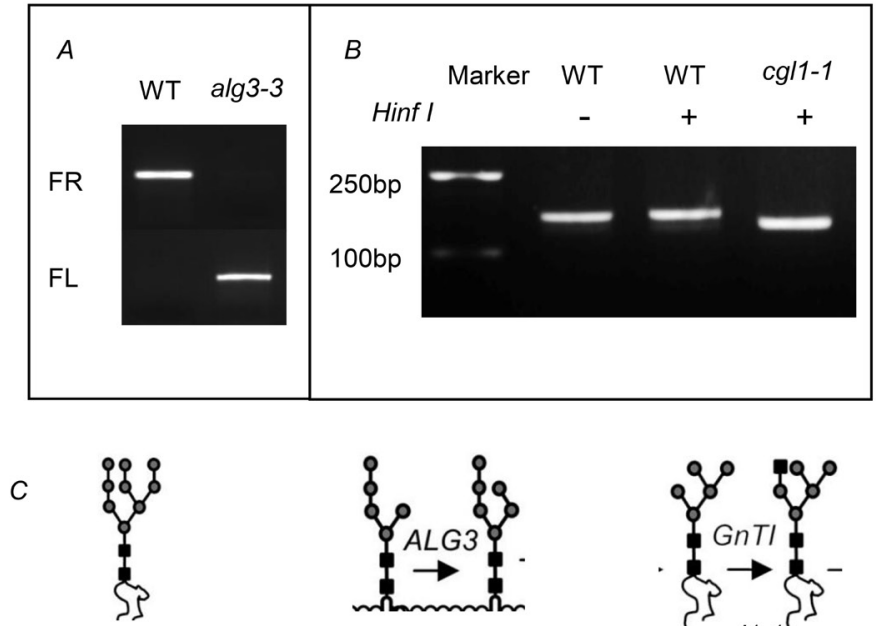

WT

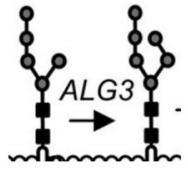

alg3-3
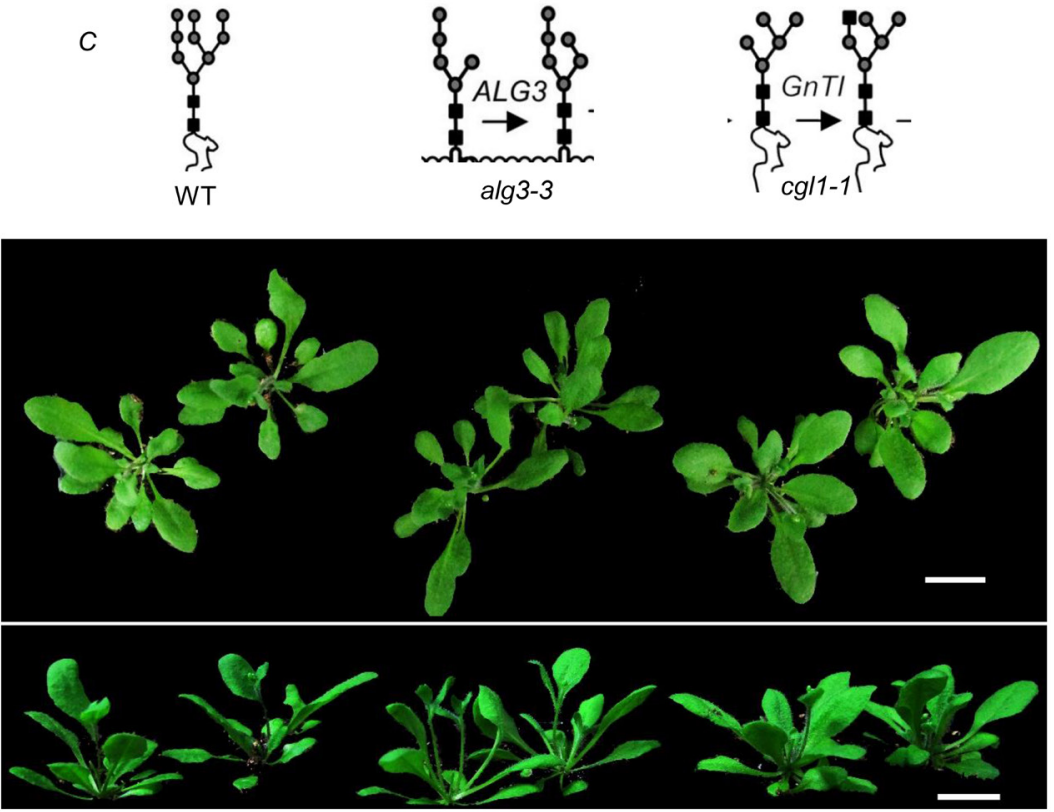

WT

alg3-3

$\operatorname{cg} / 1-1$
Fig. 1. (A) PCR analysis of Arabidopsis wild type (WT) and N-glycosylation mutants alg3-3 and cgll-1 seedlings. Genomic DNA was extracted from the leaves of alg3-3 seedlings, primers alg3-F1 (F), alg3-R1 (R), and LBa1 were used to analyze the genotypes of $\operatorname{alg} 3-3$. (B) The genotype analysis of cgl1-1, primers cgl1-dcaps-F2, and cgl1-dcaps-R2 were used for PCR, and digested by Hinf I. DNA from WT was used as positive control for the analysis. (C) Schematic representations of the major N-glycan structures of alg3-3 and cgl1-1 (top), photos of the seedlings after $21 \mathrm{~d}$ of growth in the growth chamber, scale bar $=1.0 \mathrm{~cm}$.

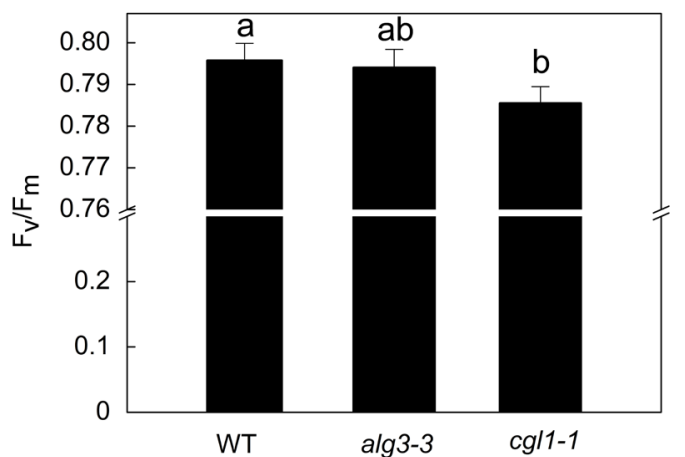

Fig. 2. The maximum quantum yield of PSII $\left(\mathrm{F}_{\mathrm{v}} / \mathrm{F}_{\mathrm{m}}\right)$ of Arabidopsis wild type (WT) and N-glycosylation mutants alg3-3 and $\mathrm{cgll}-1$. The data were statistically evaluated with one-way ANOVA multiple comparisons using Tukey's test methods. Different letters above each bar indicate significant differences at $P \leq 0.05(n=4)$.

especially, in the cgll-1 mutant (Fig. 4B). These results indicated that N-glycosylation also affected nonphotochemical processes.

$\mathrm{Y}_{(\mathrm{NO})}$ reflects the yield for other energy losses (Lazár 2015); in most cases, high $\mathrm{Y}_{(\mathrm{NO})}$ value indicates that both photochemical energy conversion and protective regulatory mechanisms are inefficient. In other words, plants have serious problems to cope with the incident radiation, either it had been already damaged or it would be photodamaged upon further irradiation (Kramer et al. 2004). Thus, contrary to previous results, the values of $\mathrm{Y}_{(\mathrm{NO})}$ showed a clear increase in the mutants, especially in cgll-1 (Fig. 4D). These results indicated that the N-glycosylation defect mutants suffered more of PSII damage than WT.

Carbonic anhydrase degradation after deglycosylation: It has been well established that carbonic anhydrase (CAH1) influences the activity of carboxylase and decarboxylase, which is essential for efficient photosynthesis capacity of the plant (Badger and Price 1994). The accurate localization and stable existence of protein is the premise of its normal physiological function. Our results showed that the stabilization of CAH1 was influenced by the treatment of tunicamycin (an antibiotic inhibiting N-glycosylation) (Fig. 5A). Our finding suggested that degradation of CAH1 might be the reason, which led to a decrease of photosynthesis in the $\mathrm{N}$-glycosylation defect mutant plants (Fig. 5B). 


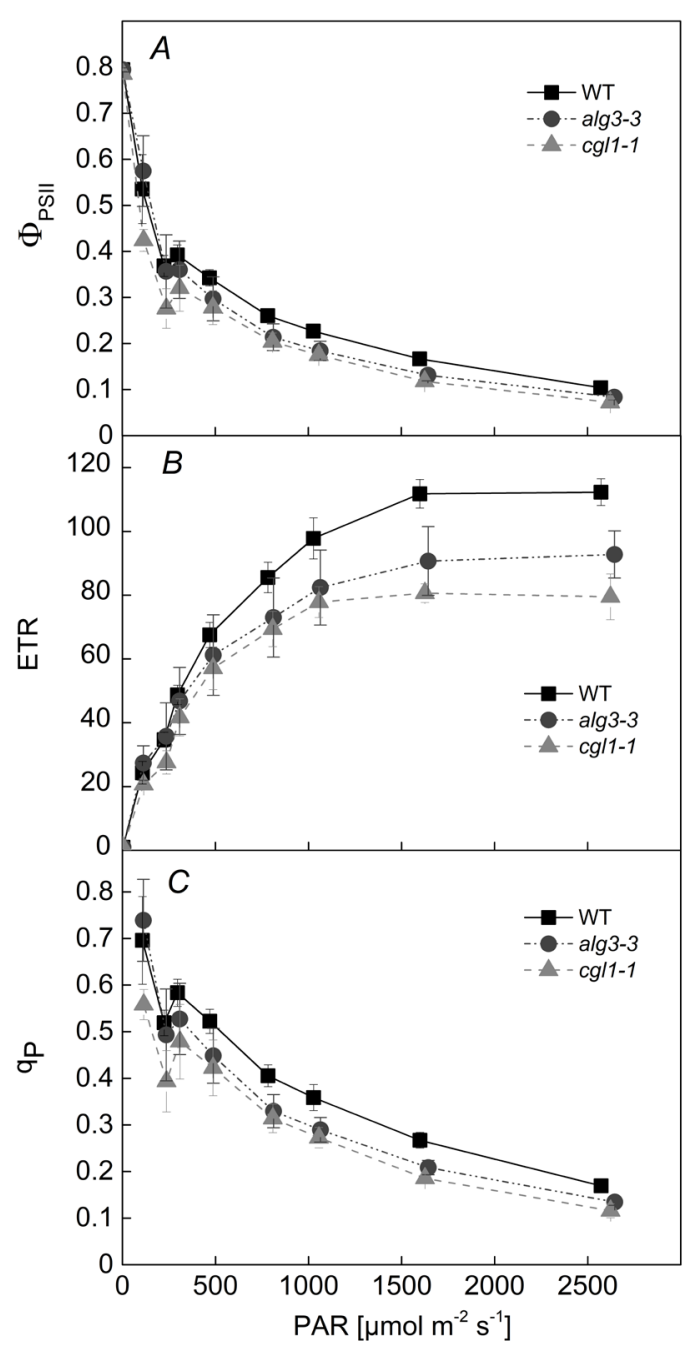

Fig. 3. The trend of the quantum yield of PSII photochemistry $\left(\Phi_{\mathrm{PSII}}\right)(A)$, the rate of photosynthetic electron transport through PSII (ETR) $(B)$, the coefficient of photochemical quenching $\left(\mathrm{q}_{\mathrm{P}}\right)$ (C) of Arabidopsis wild type (WT) and N-glycosylation mutants alg3-3 and cgll-1 with the increase of light intensity. Each curve represents an average \pm SD of 4-6 independent measurements.

\section{Discussion}

As one of the most common and essential protein modification process, $\mathrm{N}$-glycosylation plays multiple roles in eukaryotes. Previous research on this process focused on mammals or yeast, because the mutant plants exhibited rarely any visible phenotype changes under normal conditions. Recently, thousands of different N-glycosylated proteins have been identified in Arabidopsis thaliana (Zielinska et al. 2012, Song et al. 2013), indicating that glycosylation defects have also wide-ranging effects in plants. In recent years, many studies have shown that $\mathrm{Chl}$ fluorescence could represent imperceptible changes in the operation and function of the photosynthetic apparatus, even the stress tolerance of plants.

The results of the present study showed a substantial reduction of the photosynthetic capacity in the N-glycosy- lation mutant plants. However, the PSII activity, reflected by the $\mathrm{F}_{\mathrm{v}} / \mathrm{F}_{\mathrm{m}}$, seemed to be affected slightly in alg3-3 (Fig. 2). This result may suggest that in vitro measurements of PSII activity using isolated chloroplasts or thylakoid membranes might not be necessarily equivalent to PSII photochemical activity in vivo (Lu and Zhang 1998, Schansker et al. 2014). Homozygous alg3-3 and cgll-1 plants (Fig. 1A,B) did not show any visible phenotype alterations under normal laboratory conditions (Fig. 1C). However, many studies have shown that the glycosylation abnormalities result in some disturbance in the cellular level, such as the provoking of the unfolded protein responses in the ER (von Schaewen et al. 1993, Henquet et al. 2008). This was in agreement with our observation of the significant decrease of dry biomass in both alg3-3 and cgl1-1 (Fig. 1S).

The effective photochemical quantum yield of PSII $\left(\Phi_{\mathrm{PSII}}\right)$ and $\mathrm{q}_{\mathrm{P}}$ gradually decreased with the increase of light intensity (Fig. 3A,C). These values decreased much faster in the mutants compared to that in WT, while the ETR, $\mathrm{q}_{\mathrm{N}}$, and the NPQ presented a remarkable rise with increasing light intensity and the values of parameters in the mutants were lower than that of the WT at the same light intensity (Fig. 4A,B). These data indicated that capacity of capture (and transfer) of the excitation energy to the photosynthetic reaction centers decreased with increasing light intensity (Green and Parson 2003). However, with the increase of light intensity, the excess excitation energy is dissipated through nonphotochemical quenching (NPQ) of the excited state of Chl $a$ in the form of heat (Ort 2001, Demmig-Adams et al. 2014). Values of NPQ decreased in both alg3-3 and cgll-1 mutants (especially in cgll-1) compared to the WT (Fig. 4B), indicating the mutant plants may experience damage to PSII (Kozi 2006), either on the (electron) donor or the (electron) acceptor side of PSII (Pospíśil 2009, Hamdani et al. 2019).

Although plants have developed a number of photoprotection responses such as nonradiative dissipation of energy as heat at the molecular level (Müller et al. 2001, Derks et al. 2015), too much light can affect PSII and leads to damage and degradation of the reaction center (Barber and Andersson 1992). $\mathrm{Y}_{(\mathrm{NO})}$ represents basal (= nonregulated) nonphotochemical quenching, opposite to $\mathrm{Y}_{(\mathrm{NPQ})}$, which represents regulated, light-induced nonphotochemical quenching (Lazár 2015). Along with the decreased photosynthetic capacity in the mutant plants, significant growth in $\mathrm{Y}_{(\mathrm{NO})}$ was observed in alg3-3 and cgll-1 (Fig. 4D). The results intensely demonstrated that such a decrease in photochemical efficiency was at least partly associated with the significant increase of the nonphotochemical quenching, which was not photoprotective, or with photosystem damage caused by high-light stress.

Chloroplasts are organelles of endosymbiotic origin and the vast majority of chloroplast proteins are synthesized in a precursor form on cytosolic ribosomes, while a unique set of protein transport machinery is responsible for the import of the nuclear-encoded proteins into the chloroplast (Jarvis and Soll 2001, Gutensohn et al. 2006). Secretory proteins are mostly modified by $\mathrm{N}$-glycans and 


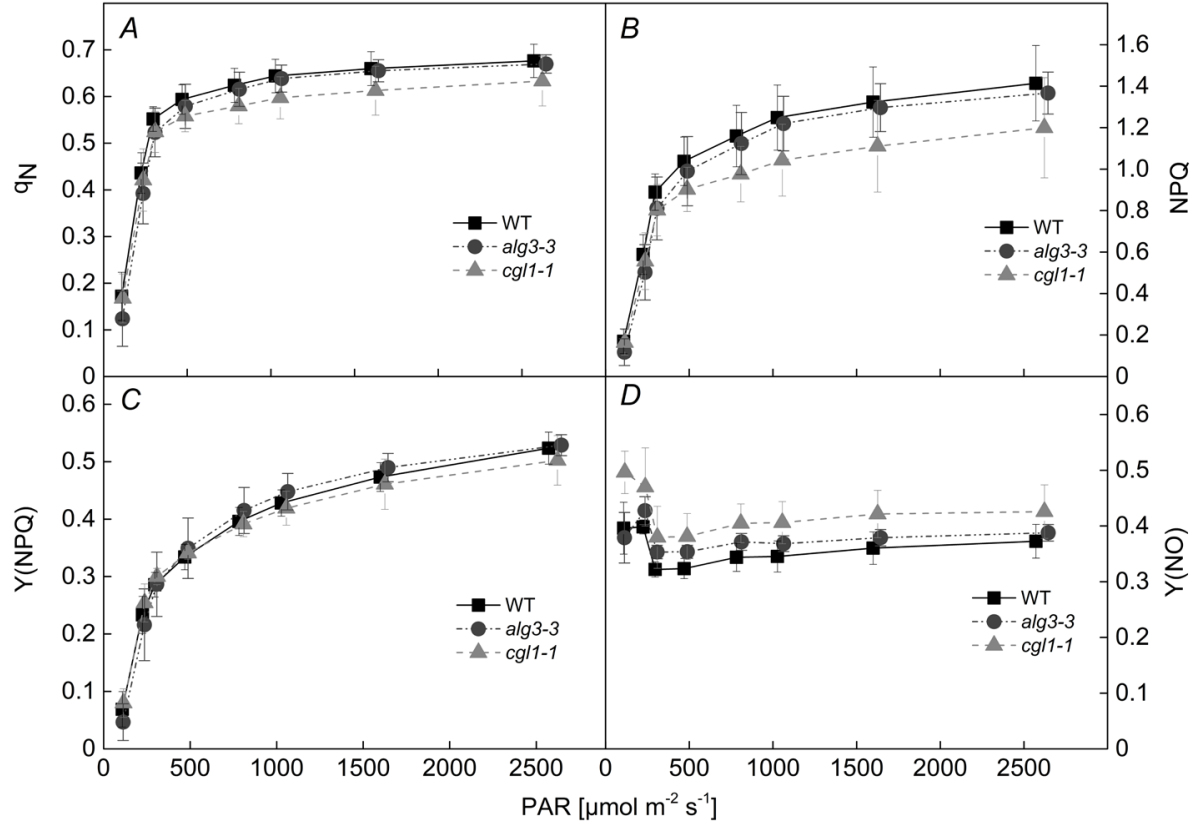

Fig. 4. Nonphotochemical chlorophyll fluorescence quenching analysis of Arabidopsis wild type (WT) and $\mathrm{N}$-glycosylation mutants alg3-3 and cgll-1. The trend of the coefficient of nonphotochemical chlorophyll fluorescence quenching $\left(\mathrm{q}_{\mathrm{N}}\right)(A)$, the NPQ parameter $(B)$, the quantum yield of regulated energy dissipation in PSII $\left(\mathrm{Y}_{(\mathrm{NPO})}\right)(C)$, and the trend of the yield for other energy losses with the increase of light intensity $\left(\mathrm{Y}_{(\mathrm{NO})}\right)(D)$. Each curve represents an average \pm SD of 4-6 independent measurements.
A

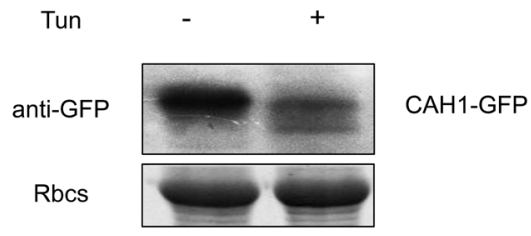

$B$
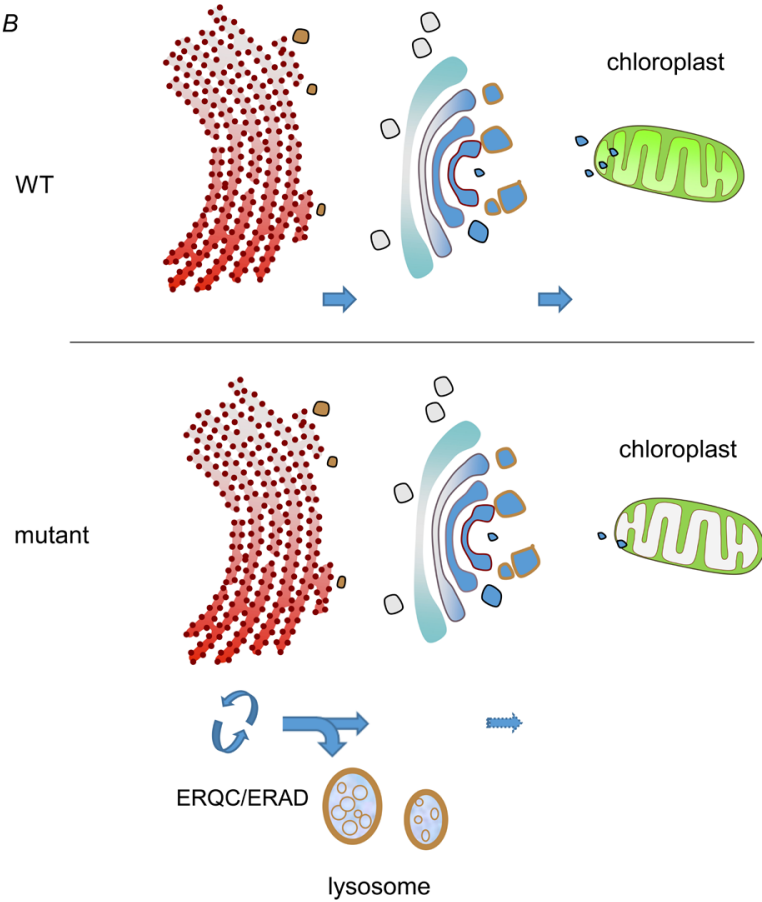

Fig. 5. (A) N-glycosylation analysis of CAH1 by migration patterns on SDS-PAGE. (B) A speculative model of CAH1 transport to the chloroplast in the $\mathrm{N}$-glycosylation mutant. $\mathrm{CAH} 1$ is synthesized on the rough ER, becomes glycosylated and transported into the chloroplast. CAH1 with abnormality of glycans resulting in the latter degradation by the ERQC/ERAD. the processing of N-glycans in the ER determines the fate of associated secretory peptides (Helenius and Aebi 2001). It is well documented that abnormal N-glycans in ER are detected by ERQC (ER-associated quality control compartment) system and misfolded proteins are removed via the ERAD (ER-associated degradation) pathway (Hong et al. 2009, Aebi 2013, Strasser 2016). Previous reports suggest that a chloroplast-located protein CAH1 becomes N-glycosylated before entering the chloroplast through the secretory pathway (Villarejo et al. 2005, Burén et al. 2011), indicating that N-glycosylation plays the role in regulation of secretory pathway for chloroplast-located proteins. The well N-glycosylated $\mathrm{CAH} 1$ is transported into the chloroplast through the secretory pathway in the WT plants and then participates in the process of photosynthesis. On the other hand, the misfolded or damaged CAH1 (caused by perturbation of $\mathrm{N}$-glycosylation) in the mutant plants might activate the ERQC/ERAD machinery, which may finally lead to the degradation of CAH1 before entering the chloroplast (Fig. 5B). In addition, CAH1 degraded obviously after deglycosylation by tunicamycin (Fig. $5 A$ ). These findings suggested that the decline of photosynthetic capacity in the alg3-3 or cgll-1 mutant may be also a consequence of the functional decomposition of CAH1 caused by $\mathrm{N}$-glycosylation defects. Moreover, it is worth mentioning that the calculated significant decrease in dry biomass of alg3-3 and cgll-1 (Fig. 1S) was thus consistent with the findings of the reduced photosynthetic rate, although the Chl content barely changed (Fig. 2S, supplement).

$\mathrm{N}$-glycosylation is essential for multicellular life and its complete absence is embryonically lethal. In this work, we found that the obviously decreased photosynthetic capacity in N-glycosylation defective mutants alg3-3 and cgl1-1 might be associated with the disturbance of the PSII and impaired electron transfer system. Based on the above results, we also showed that $\mathrm{N}$-glycosylation 
regulated the photosynthetic capacity of plants at least in part by CAH1, although the exact mechanism(s) must be confirmed. In conclusion, $\mathrm{N}$-glycosylation is required for plants to maintain the normal function of photosynthesis.

\section{References}

Aebi M.: N-linked protein glycosylation in the ER. - BBA-Mol. Cell. Res. 1833: 2430-2437, 2013.

Aebi M., Bernasconi R., Clerc S., Molinari M.: N-glycan structures: recognition and processing in the ER. - Trends Biochem. Sci. 35: 74-82, 2010.

Badger M.R., Price G.D.: The role of carbonic-anhydrase in photosynthesis. - Annu. Rev. Plant Phys. 45: 369-392, 1994.

Barbagallo R.P., Oxborough K., Pallett K.E., Baker N.R.: Rapid, noninvasive screening for perturbations of metabolism and plant growth using chlorophyll fluorescence imaging. - Plant Physiol. 132: 485-493, 2003.

Barber J., Andersson B.: Too much of a good thing: light can be bad for photosynthesis. - Trends Biochem. Sci. 17: 61-66, 1992.

Bickel T., Lehle L., Schwarz M. et al.: Biosynthesis of lipidlinked oligosaccharides in Saccharomyces cerevisiae: Alg13p and $A \lg 14 \mathrm{p}$ form a complex required for the formation of GlcNAc(2)-PP-dolichol. - J. Biol. Chem. 280: 34500-34506, 2005.

Burén S., Ortega-Villasante C., Blanco-Rivero A. et al.: Importance of post-translational modifications for functionality of a chloroplast-localized carbonic anhydrase (CAH1) in Arabidopsis thaliana. - PLoS ONE 6: e21021, 2011.

Demmig-Adams B., Adams III W.W., Barker D.H. et al.: Using chlorophyll fluorescence to assess the fraction of absorbed light allocated to thermal dissipation of excess excitation. Physiol. Plantarum 98: 253-264, 1996.

Demmig-Adams B., Garab G., Adams III WW., Govindjee: NonPhotochemical Quenching and Energy Dissipation in Plants, Algae and Cyanobacteria. Pp. 649. Springer, Dordrecht 2014.

Derks A., Schaven K., Bruce D.: Diverse mechanisms for photoprotection in photosynthesis. Dynamic regulation of photosystem II excitation in response to rapid environmental change. - BBA-Bioenergetics 1847: 468-485, 2015.

Dyballa N., Metzger S.: Fast and sensitive colloidal coomassie G-250 staining for proteins in polyacrylamide gels. - JOVE-J. Vis. Exp. 3: 422-427, 2009.

Genty B., Briantais J.M., Baker N.R.: The relationship between the quantum yield of photosynthetic electron-transport and quenching of chlorophyll fluorescence. - BBA-Gen. Subjects 990: 87-92, 1989.

Green B., Parson W.W.: Light-Harvesting Antennas in Photosynthesis. Pp. 516. Springer, Dordrecht 2003.

Gutensohn M., Fan E.G., Frielingsdorf S. et al.: Toc, Tic, Tat et al:: Structure and function of protein transport machineries in chloroplasts. - Plant Physiol. 163: 333-347, 2006.

Hamdani S., Khan N., Perveen S. et al: Changes in the photosynthesis properties and photoprotection capacity in rice (Oryza sativa) grown under red, blue, or white light. Photosynth. Res. 139: 107-121, 2019.

Harbinson J., Genty B., Baker N.: The relationship between $\mathrm{CO}_{2}$ assimilation and electron transport in leaves. - Photosynth. Res. 25: 213-224, 1990.

Helenius A., Aebi M.: Intracellular functions of N-linked glycans. - Science 291: 2364-2369, 2001.

Henquet M., Lehle L., Schreuder M. et al.: Identification of the gene encoding the $\alpha 1,3$-mannosyltransferase (ALG3) in Arabidopsis and characterization of downstream N-glycan processing. - Plant Cell 20: 1652-1664, 2008.

Higgins C.F.: Flip-flop: The transmembrane translocation of lipids. - Cell 79: 393-395, 1994.

Hong Z., Jin H., Fitchette A.-C. et al.: Mutations of an $\alpha 1,6$ mannosyltransferase inhibit endoplasmic reticulum-associated degradation of defective brassinosteroid receptors in Arabidopsis. - Plant Cell 21: 3792-3802, 2009.

Jarvis P., Soll J: Toc, Tic, and chloroplast protein import. - BBAMol. Cell. Res. 1541: 64-79, 2001.

Kang B.S., Baek J.H., Macoy D.M. et al.: N-glycosylation process in both ER and Golgi plays pivotal role in plant immunity. - J. Plant Biol. 58: 374-382, 2015.

Kang J.S., Frank J., Kang C.H. et al.: Salt tolerance of Arabidopsis thaliana requires maturation of $\mathrm{N}$-glycosylated proteins in the Golgi apparatus. - P. Natl. Acad. Sci. USA 105: 5933-5938, 2008.

Koiwa H., Li F., McCully M.G. et al.: The STT3a subunit isoform of the Arabidopsis oligosaccharyltransferase controls adaptive responses to salt/osmotic stress. - Plant Cell 15: 2273-2284, 2003.

Kozi A.: Production and scavenging of reactive oxygen species in chloroplasts and their functions. - Plant Physiol. 141: 391396, 2006.

Kramer D.M., Johnson G., Kiirats O., Edwards G.E.: New fluorescence parameters for the determination of $\mathrm{Q}_{\mathrm{A}}$ redox state and excitation energy fluxes. - Photosynth. Res. 79: 209, 2004.

Kupriyanova E., Pronina N., Los D.: Carbonic anhydrase a universal enzyme of the carbon-based life. - Photosynthetica 55: 3-19, 2017.

Larkin A., Imperiali B.: The expanding horizons of asparaginelinked glycosylation. - Biochemistry 50: 4411-4426, 2011.

Lazár D.: Parameters of photosynthetic energy partitioning. J. Plant Physiol. 175: 131-147, 2015.

Liang Y., Urano D., Liao K.L. et al.: A nondestructive method to estimate the chlorophyll content of Arabidopsis seedlings. Plant Methods 13: 26, 2017.

Liu C., Niu G., Zhang H. et al.: Trimming of N-glycans by the Golgi-localized $\alpha-1,2$-mannosidases, MNS1 and MNS2, is crucial for maintaining RSW2 protein abundance during salt stress in Arabidopsis. - Mol. Plant 11: 678-690, 2018.

Lu C., Zhang J.: Modifications in photosystem II photochemistry in senescent leaves of maize plants. - J. Exp. Bot. 49: 16711679, 1998.

Magyar M., Sipka G., Kovács L. et al.: Rate-limiting steps in the dark-to-light transition of Photosystem II-revealed by chlorophyll- $a$ fluorescence induction. - Sci Rep.-UK 8: 2755, 2018.

McCormack M.E., Liu X., Jordan M.R., Pajerowska-Mukhtar K.M.: An improved high-throughput screening assay for tunicamycin sensitivity in Arabidopsis seedlings. - Front. Plant Sci. 6: 663, 2015.

Müller P., Li X.P., Niyogi K.K.: Non-photochemical quenching. A response to excess light energy. - Plant Physiol. 125: 15581566, 2001.

Murchie E.H., Lawson T.: Chlorophyll fluorescence analysis: A guide to good practice and understanding some new applications. - J. Exp. Bot. 64: 3983-3998, 2013.

Ort D.R.: When there is too much light. - Plant Physiol. 125: 29-32, 2001.

Pospíśil P.: Production of reactive oxygen species by photosystem II. - BBA-Bioenergetics 1787: 1151-1160, 2009.

Reiss G., te Heesen S., Zimmerman J. et al.: Isolation of the $A L G 6$ locus of Saccharomyces cerevisiae required for glucosylation in the N-linked glycosylation pathway. - Glycobiology 6: 493-498, 1996. 
Samuelsson G., Karlsson J.: Chloroplastic carbonic anhydrases. In: Aro E.-M., Andersson B. (ed.): Regulation of Photosynthesis. Pp. 313-320. Springer, Dordrecht 2001.

Schansker G., Tóth S.Z., Holzwarth A.R., Garab G.: Chlorophyll $a$ fluorescence: Beyond the limits of the $\mathrm{Q}_{\mathrm{A}}$ model. - Photosynth. Res. 120: 43-58, 2014.

Schoberer J., Shin Y.J., Vavra U. et al.: Analysis of protein glycosylation in the ER. - In: Hawes C., Kriechbaumer V. (ed.): The Plant Endoplasmic Reticulum. Methods in Molecular Biology. Vol. 1691. Pp. 205-222. Humana Press, New York 2018.

Schollen E., Grünewald S., Keldermans L. et al.: CDG-Id caused by homozygosity for an ALG3 mutation due to segmental maternal isodisomy UPD3(q21.3-qter). - Eur. J. Med. Genet. 48: 153-158, 2005.

Silberstein S., Gilmore R.: Biochemistry, molecular biology, and genetics of the oligosaccharyltransferase. - FASEB J. 10: 849-858, 1996.

Song W., Mentink R.A., Henquet M.G. et al.: N-glycan occupancy of Arabidopsis N-glycoproteins. - J. Proteomics 93: 343-355, 2013.

Stagljar I., te Heesen S., Aebi M.: New phenotype of mutations deficient in glucosylation of the lipid-linked oligosaccharide: cloning of the ALG8 locus. - P. Natl. Acad. Sci. USA 91:
5977-5981, 1994.

Stirbet A.: Excitonic connectivity between photosystem II units: What is it, and how to measure it? - Photosynth. Res. 116: 189-214, 2013.

Strasser R.: Plant protein glycosylation. - Glycobiology 26: 926939, 2016.

Villarejo A., Burén S., Larsson S. et al.: Evidence for a protein transported through the secretory pathway en route to the higher plant chloroplast. - Nat. Cell Biol. 7: 1224-1231, 2005.

von Schaewen A., Sturm A., O'Neill J., Chrispeels M.J.: Isolation of a mutant Arabidopsis plant that lacks N-acetyl glucosaminyl transferase I and is unable to synthesize Golgimodified complex N-linked glycans. - Plant Physiol. 102: 1109-1118, 1993.

Zhang M., Henquet M., Chen Z. et al.: LEW3, encoding a putative $\alpha$-1,2-mannosyltransferase (ALG11) in N-linked glycoprotein, plays vital roles in cell-wall biosynthesis and the abiotic stress response in Arabidopsis thaliana. - Plant J. 60: 983-999, 2009

Zielinska D.F., Gnad F., Schropp K. et al.: Mapping N-glycosylation sites across seven evolutionarily distant species reveals a divergent substrate proteome despite a common core machinery. - Mol. Cell 46: 542-548, 2012.

(C) The authors. This is an open access article distributed under the terms of the Creative Commons BY-NC-ND Licence. 